\title{
The Process of Coagulation in Smoke*
}

$I^{N}$ contradistinction to hydrosols, smokes are unstable systems. Their particles cohere when brought together by Brownian agitation, and this process of coagulation proceeds spontaneously until the system becomes a coarse suspension of complex aggregates, and finally sediments rapidly.

In recent years, special methods have been developed for counting the number of particles in many types of smoke, and the process of coagulation has been studied quantitatively in a variety of systems. It has been found experimentally that the decrease with time of the number of particles in a smoke is given to a first approximation by the expression $1 / n-1 / n_{0}=K t$, where $n$ is the number of particles present in a given volume at time $t, n_{0}$ the initial number and $K$ a constant. As is well known, an expression of the same form is valid for the recombination of ions, but in a normal smoke the combination of particles to form aggregates is only influenced slightly, if at all, by electrical charges.

A comparison of the coagulation constants or $K$ values for different smokes of about the same mass concentration shows that they vary between comparatively narrow limits from about $0.8 \times 10^{-9} \mathrm{~cm}^{3}$ sec. $^{-1}$ in the case of oxide smokes formed in the electric arc to $0.50 \times 10^{-9} \mathrm{~cm}^{3} \mathrm{sec}^{-1}$ for a standard smoke of stearic acid of mass concentration of $15 \mathrm{mgm}$. per cubic metre.

The structure of the aggregates and the nature of the material do not, so far as is known at present, exert any marked effect on the rate of coagulation, which appears to be a purely physical process dependent on the chance encounter of particles in Brownian motion. Experimental evidence, however, shows that the coagulation constant increases rapidly when the average size of particle falls below $1 \times 10^{-5} \mathrm{~cm}$. radius, and it increases also with the degree of heterogeneity of the smoke. By a careful study of the conditions under which a smoke is formed, Patterson and Cawood have prepared disperse systems of stearic acid particles which initially approach to uniformity in size. This was accomplished by dispersing the heated solid in a rapid

* Substance of the Liversidge Lecture delivered by Prof. $R$. Whytlaw-Gray, O.B.E., F.R.S., before the Chemical Society on February 14. blast of hot air, and by this means diluting rapidly the concentrated smoke before coagulation had proceeded far. Such smokes are readily reproducible and form standard systems which coagulate at the same rate and contain the same number of particles, and since they form compact aggregates they approximate in character to ideal systems of spherical particles.

The well-known theory of von Smoluchowski, which has been confirmed experimentally for the coagulation of sols by the comprehensive researches of Tuorila, when modified so as to apply to aerial systems, enables the coagulation constant of a homogeneous smoke to be calculated from first principles. Patterson and Cawood have shown that when the experimental data for these 'blown smokes' are interpreted rightly, a remarkably close agreement between theory and experiment is obtained. Theory also indicates that whilst in sols undergoing quick coagulation the rate should be independent of size, in aerial systems it should increase as the particle size diminishes; a prediction in entire conformity with experiment.

Both in sols and aerosols, theory shows that heterogeneity must increase the chances of encounter between particles, but although in sols it has been possible to check experimentally the extension of von Smoluchowski's theory proposed by H. Müller, for heterogeneous smokes theory so far has not proved more than a qualitative guide.

The study then of smokes affords strong confirmatory evidence of the validity of Smoluchowski's theory, and lends support to the view that this continuous process so characteristic of these systems is akin to the quick coagulation of sols in the presence of electrolytes, and points to the probability that in both classes of system every collision between particles is effective. It must, however, be noted that the coagulation constants for systems of fine suspensions in water and in air are widely different, the latter being about a hundred times as great as the former. But since the time taken to reduce the original number of particles in a system to any given fraction depends on the number as well as on $K$, the disappearance of particles by coagulation in town fogs and other polluted atmospheres will be slow.

\section{British Industries Fair, I935}

$\mathrm{I}^{\mathrm{N}}$ the account given last year in NATURE of the 1934 British Industries Fair, it was described as the largest national trade fair in the world. The 1935 Fair, which opened in London on February 18, is even larger than its immediate predecessor-a reflection, it may be hoped, of increasing prosperity in the nation's trade. At Olympia the lighter industries occupy every available square foot of exhibiting space, while the textile and furnishing sections at the White City are larger and more fully representative than ever. The main object of the Fair is, of course, a commercial one ; the most welcome visitors are buyers. But the Fair has, undoubtedly, an educational value, for it presents to the visitor, in an attractive and accessible form and in a condensed space, a general survey of the results of the nation's manufacturing industry. Moreover, every changing phase in the tastes and habits of the people is reflected in such a collection of manufactured articles as is to be found at the Fair. The removal of much that is tedious and unnecessary from domestic work is indicated by the increased popularity of chromiumplated ware and of stainless steel and stainless silver articles, and by the space allotted in the Fair to the exhibition of devices for domestic mechanisation. The exhibition in larger quantities of open-air equipment of all kinds indicates that increasingly the leisure of the people is being used in healthy pursuits. At Olympia, the exhibition is, as previously, divided into sections according to industries, and it is perhaps symbolic of the increasing recognition of the co-operation which must exist between science and industry, that the section devoted to the exhibition of the products of the Scientific and Optical 